DOI: $10.12957 /$ geouerj.2013.6912

\title{
SIMULACIÓN DE CAIIDA DE CENIZA DEL VOLCÁN IRAZÚ APLICANDO EL PROGRAMA TEPHRA MODIFICADO
}

\section{IRAZÚ VOLCANO ASH FALL SIMULATION USING A MODIFICATION OF THE TEPHRA SOFTWARE}

\author{
Gustavo Barrantes Castillo \\ Escuela de Ciencias Geográficas, Universidad Nacional. Costa Rica \\ gbarran@una.ac.cr \\ Santiago Núñez Corrales \\ Dirección de Tecnología, Ministerio de Ciencia y Tecnología. Costa Rica \\ snunezcr@gmail.com \\ José Castro Mora \\ Escuela de Ingeniería en Computación, Instituto Tecnológico de Costa Rica \\ jose.r.castro@gmail.com \\ Eduardo Malavassi Rojas \\ Jubilado, Observatorio Vulcanológico y Sismológico de Costa Rica, Universidad Nacional \\ emalavassi@gmail.com \\ Casia Soto Montoya \\ Escuela de Ingeniería Forestal, Instituto Tecnológico de Costa Rica \\ cassiaspp@gmail.com
}

\section{Resumen}

El presente trabajo utiliza el modelo de Suzuki (Suzuki, 1983) para simular la caída de ceniza volcánica en la versión TEPHRA (Connors, 1991). El programa TEPHRA modificado es sometido a prueba al simular exitosamente la distribución de ceniza de los eventos eruptivos mejor conocidos en el volcán Irazú, Costa Rica. Adicionalmente, la masa de ceniza acumulada por 52 simulaciones de diseño concuerda satisfactoriamente con la distribución de las isopacas de la ceniza depositada durante el periodo 1963-65 del volcán Irazú y relativamente bien con la distribución de isopacas de la ceniza depositada durante los últimos 2600 años de actividad. Esta situación justifica la aplicación del programa TEPHRA modificado en la elaboración de mapas de amenaza por caída de ceniza para el Volcán Irazú y otros volcanes en los cuales se tenga apropiada información sobre dirección del viento y características eruptivas más probables de presentarse en el volcán.

Palabras Clave: Caída de ceniza, simulación computacional, mapas de amenaza, TEPHRA, escenarios de riesgo, Volcán Irazú.

Geo UERJ - Ano $15, n^{\circ} .24$, v. $1,1^{\circ}$ semestre de 2013 p. 5-17

ISSN: $1415-7543 E-I S S N$ : $1981-9021$

http://www.e-publicacoes.uerj.br/index.php/geouerj 


\begin{abstract}
This paper uses the Suzuki volcanic ash simulation model (Suzuki, 1983) to simulate volcanic ashfall in the tephra version TEPHRA (Connors, 1991). The modified TEPHRA volcanic ash simulation program has been used to develop computer simulations of volcanic ashfall produced by Irazú volcano, Costa Rica. The ability of the modified TEPHRA program to simulate volcanic ashfalls is tested by reproducing successfully the ash fall distribution of the best known eruptive episodes from the 1963-1965 active period. Additionally, the mass of accumulated ash during 52 simulations agrees very well with the ash isopachs deposited during the 1963-1965 active period and well with the distribution of ash isopachs deposited during the last 2600 years. We conclude that the modified TEPHRA program is suitable to prepare ash fall hazards maps for Irazú and other volcanoes, on the condition that there is good knowledge of their most likely eruptive characteristics and local wind direction.
\end{abstract}

Key words: Ashfall, computer simulations, TEPHRA, hazards maps, risk scenarios, Irazú volcano.

\title{
INTRODUCCION
}

En los últimos 30 años, la modelación física de los procesos volcánicos ha cobrado relevancia como un mecanismo para simular los efectos de una erupción y de esta forma comprender sus posibles impactos en el ambiente (Felpeto, 2002). Los modelos conceptuales creados a partir del conocimiento de la física del problema pueden ser traducidos en conjuntos de ecuaciones matemáticos, secuencias de reglas explícitas que convierten entradas numéricas en salidas numéricas con un propósito específico (Dingman, 2008). Los modelos matemáticos de procesos naturales son una representación parcial de la realidad, que reproducen algunas características de la realidad de manera selectiva, consideradas relevantes para el problema en cuestión.

La simulación computacional corresponde a la implementación de un modelo matemático que describe la dinámica esencial del comportamiento de un sistema de interés. Su utilidad fundamental está en la posibilidad de diseñar y realizar experimentos computacionales con el modelo y extraer conclusiones relevantes a partir de sus resultados. La simulación permite examinar el comportamiento del sistema en casos en los que resulta imposible o impráctico ejecutar el experimento o esperar a que suceda en la realidad.

Por otra parte, la simulación es una aplicación natural para computación de alto rendimiento y para computación paralela distribuida, con lo cual se logra una fuerte sinergia entre aplicaciones

Geo UERJ - Ano $15, n^{\circ} .24$, v. $1,1^{\circ}$ semestre de 2013 p. $5-17$

ISSN: 1415-7543E-ISSN: 1981-9021

http://www.e-publicacoes.uerj.br/index.php/geouerj 
y arquitecturas computacionales orientadas a procesamiento numérico masivo (Barrantes et al., 2007). Generalmente su diseño busca un equilibrio entre la precisión y el costo computacional. La importancia de aplicar esta tecnología al problema de la caída de ceniza en Costa Rica radica en la posibilidad de anticipar posibles áreas y grados de afectación, por medio de la técnica del escenario. En la estimación del riesgo el análisis de escenarios permite la identificación y priorización de potenciales desastres, o al menos sus dos casos más relevantes denominados: "el peor escenario probable" y el "escenario más probable" (Subsecretaría de Desarrollo Regional y Administrativa de Chile (2011). En el presente artículo se evaluará la aplicabilidad del programa TEPHRA modificado, a la amenaza que representa la caída de ceniza proveniente del Volcán Irazú.

\section{EL PROGRAMA TEPHRA}

Actualmente existen varios programas para simular la caída de ceniza tales como: FALL3D, HAZMAP y TEPHRA. Cada uno de ellos cuenta con diferentes niveles de complejidad (y por consiguiente de precisión) tanto en la cantidad de parámetros de entrada, como en la complejidad computacional inherente a cada programa (Scollo et al 2008).

A pesar de que TEPHRA no contempla la topografía ni la agregación de partículas, se ha seleccionado por su fácil aplicación y su menor costo computacional. Adicionalmente, en su comparación con otros modelos semi-analíticos y 3D, Scollo et al (2008) señala que la diferencia entre modelos simples y complejos es comparable a la incertidumbre que típicamente producen las entrada en los modelos complejos, de esta manera, a menos que se realice una apropiada obtención de dichas entradas, el uso de modelos complejos no implicar una ventaja particular en la realización de pronósticos.

TEPHRA es un programa de simulación computacional que implementa el modelo Suzuki (Suzuki, 1983), en el cual la difusión, advección y sedimentación son calculadas mediante una ecuación de conservación de masa (Bonadonna et al., 2005). La primera versión del software fue implementada por Connor (2001) y posteriormente modificada y extendida por un grupo de investigación multidisciplinario para su implementación en el Volcán Irazú (Núñez, Barrantes, Malavassi, \& Brenes, 2010).

Geo UERJ - Ano $15, n^{\circ} .24$, v. $1,1^{\circ}$ semestre de 2013 p. $5-17$

ISSN: $1415-7543 E-I S S N$ : $1981-9021$

http://www.e-publicacoes.uerj.br/index.php/geouerj 
El modelo se basa en una ecuación de continuidad que describe la variación espacio-temporal de la concentración de partículas C en la atmósfera [1] (Armienti \& Macedonio, 1988):

$$
\frac{\partial \mathrm{C}}{\partial \mathrm{t}}+u \frac{\partial \mathrm{C}}{\partial \mathrm{x}}+v \frac{\partial \mathrm{C}}{\partial \mathrm{y}}+\frac{\partial\left(V_{S} \mathrm{C}\right)}{\partial z}=K_{x} \frac{\partial^{2} C}{\partial_{x^{2}}}+K_{y} \frac{\partial^{2} C}{\partial_{y^{2}}}+K_{z} \frac{\partial^{2} C}{\partial_{z^{2}}}+S
$$

donde

$$
K_{x} \frac{\partial^{2} C}{\partial_{x^{2}}}+K_{y} \frac{\partial^{2} C}{\partial_{y^{2}}}+K_{z} \frac{\partial^{2} C}{\partial_{z^{2}}}
$$

es el término de difusión; $K_{x}, K_{y}, K_{z}$ son los coeficientes de difusión turbulenta en las direcciones $x, y, z$.

$$
u \frac{\partial \mathrm{C}}{\partial \mathrm{x}}+v \frac{\partial \mathrm{C}}{\partial \mathrm{y}}
$$

es el término de advección según el campo de vientos horizontales $u, v$.

$$
\frac{\partial\left(V_{S} \mathrm{C}\right)}{\partial z}
$$

es el término de depositación; donde v es la velocidad límite de caída de las partículas.

Las entradas del programa TEPHRA son: coordenadas y elevación de la fuente; masa total; tamaño, forma y densidad de las partículas; altura de la columna; velocidad inicial de la erupción; velocidad y dirección del viento.

Una función de densidad de probabilidad es utilizada para describir la difusión de la tefra fuera de la columna en la atmósfera circundante, donde es posible el transporte por el viento. En su formulación se utiliza el parámetro $\beta$, el cual controla la distribución en altura de la concentración de ceniza. Altos valores de $\beta$ resultan en una mayor proporción de tefra presente en la parte alta de la columna eruptiva. La velocidad ascendente de las partículas decrece proporcionalmente con la altura en función de la altura elevada a la potencia $\lambda$, a la cual Connor asigna el valor de 1 (Connor et al., 2001). De esta manera, la incertidumbre del modelo crece con la incorporación de estos dos parámetros, aumentando la necesidad de su calibración. Una completa descripción del programa puede consultarse en Connor (2002).

\section{SIMULACIÓN DE CAÍDA DE CENIZA PARA EL VOLCÁN IRAZÚ}


Con la finalidad de evaluar la aplicabilidad del programa TEPHRA en Costa Rica, tanto en la creación de escenarios de interés como en la elaboración de mapas probabilísticos, se procedió a seleccionar un volcán que tuviese registros históricos, estudios geológicos, tefrocronológicos, así como un episodio eruptivo, suficientemente documentados. El volcán que mejor se aproximó a estos requerimientos fue el Irazú.

Entre 1963-65 ocurrió un episodio eruptivo en el Volcán Irazú, que afectó severamente un área de $100 \mathrm{~km}^{2}$ (que incluyó las poblaciones siguientes: Coronado, Montes de Oca, Curridabat, Llano grande, San Juan de Concepción, Dulce Nombre, Potrero Cerrado y parcialmente a Desamparados y Goicoechea) y de manera importante un área de $300 \mathrm{~km}^{2}$. Cuyas pérdidas totales fueron estimadas en 2.000.000 dólares (Escuela Centroamericana de Geología, 1993). En aquella época los principales daños directos correspondieron a la pérdida de cultivos (como café, papa, maíz, tomate), ganado, producción lechera, así como los problemas asociados con la acumulación de la ceniza en las ciudades próximas al volcán, situación que llevo a que la Ciudad de San José (una de las más afectadas), fuese conocida como la ciudad de las escobas (Armbrister, 1964), de manera indirecta provocó la ocurrencia de varios lahares o corrientes de barro que descendieron por los principales cauces que drenan el cono del Irazú, uno de los cuales cobró la vida de 20 personas y destruyó alrededor de 300 viviendas (Waldron, 1967).

El área de afectación del período activo 63-65 corresponde, hoy día, con un porcentaje importante de la llamada el Gran Área Metropolitana (GAM). Mientras que en aquella época la población del Costa Rica rondaba los 1.2 millones de habitantes, en su mayoría población rural, hoy la población de Costa Rica supera los 4.3 millones de habitantes y es precisamente en el GAM donde se ha experimentado el mayor proceso de urbanización, que concentra más del $60 \%$ de la población total del país y alrededor del $75 \%$ de todas las actividades económicas productivas (Astorga, 2008). Estas cifras resaltan la importancia de contar con herramientas que faciliten una gestión de riesgo y la planificación territorial, ante la amenaza por caída de ceniza. La simulación de caída de tefra para el Volcán Irazú fue posible gracias a la plataforma instalada por el proyecto Cluster Interuniversitario (Gómez, 2008), específicamente del Cluster de la Universidad Nacional (UNA).

En la implementación de la simulación para el caso del Volcán Irazú, se utilizó una rejilla uniforme con una resolución horizontal de $1 \mathrm{Km}$, que abarca un área total de $130.000 \mathrm{~km}^{2}$, 
localizada sobre el Valle Central de Costa Rica. Los parámetros de entrada relacionados con las partículas de ceniza fueron deducidos del trabajo de Alvarado (1993) y ajustados por un previo análisis de sensibilidad del modelo. Las alturas de la columna eruptiva se tomaron de los reportes presentados por Murata et al., (1965) y Barquero (1977) y de la revisión de los periódicos de la época. La masa total $M$ se estimó a partir de la ley de potencia empírica:

$$
M=\rho\left(\frac{H}{1670}\right)^{4} \tau
$$

Donde $H$ es la altura de columna, $\rho$ es la densidad media de la tefra y $\tau$ es la duración sostenida de la face eruptiva.

Finalmente, los parámetros de entrada relacionados con el viento fueron deducidos a partir del trabajo de Alvarado \& Fernández (2001) al no exitir registros de vientos de la época.

\subsection{ESCENARIOS INDIVIDUALES}

De las recopilaciones históricas disponibles (Barquero, 1977; Murata et al., 1965; Clark, 1993; Escuela Centroamericana de Geología, 1993), se escogieron los escenarios que disponían de más información para realizar a una calibración, sin embargo la ausencia de datos sobre los volúmenes y grosores de la tefra depositada en cada evento, solo hizo posible realizar una calibración cualitativa a partir de la comparación entre las áreas y grados de afectación reportados para cada evento, con la distribución de la tefra estimada por medio de la simulación. Los escenarios seleccionados fueron los del 22 y el 23 de marzo; el 17, 20, 23 y 25 de abril; 23 de octubre; 1 el diciembre, todos correspondientes al año 1963. Por razones espacio solo se analizarán cuatro escenarios (Figura 1). 


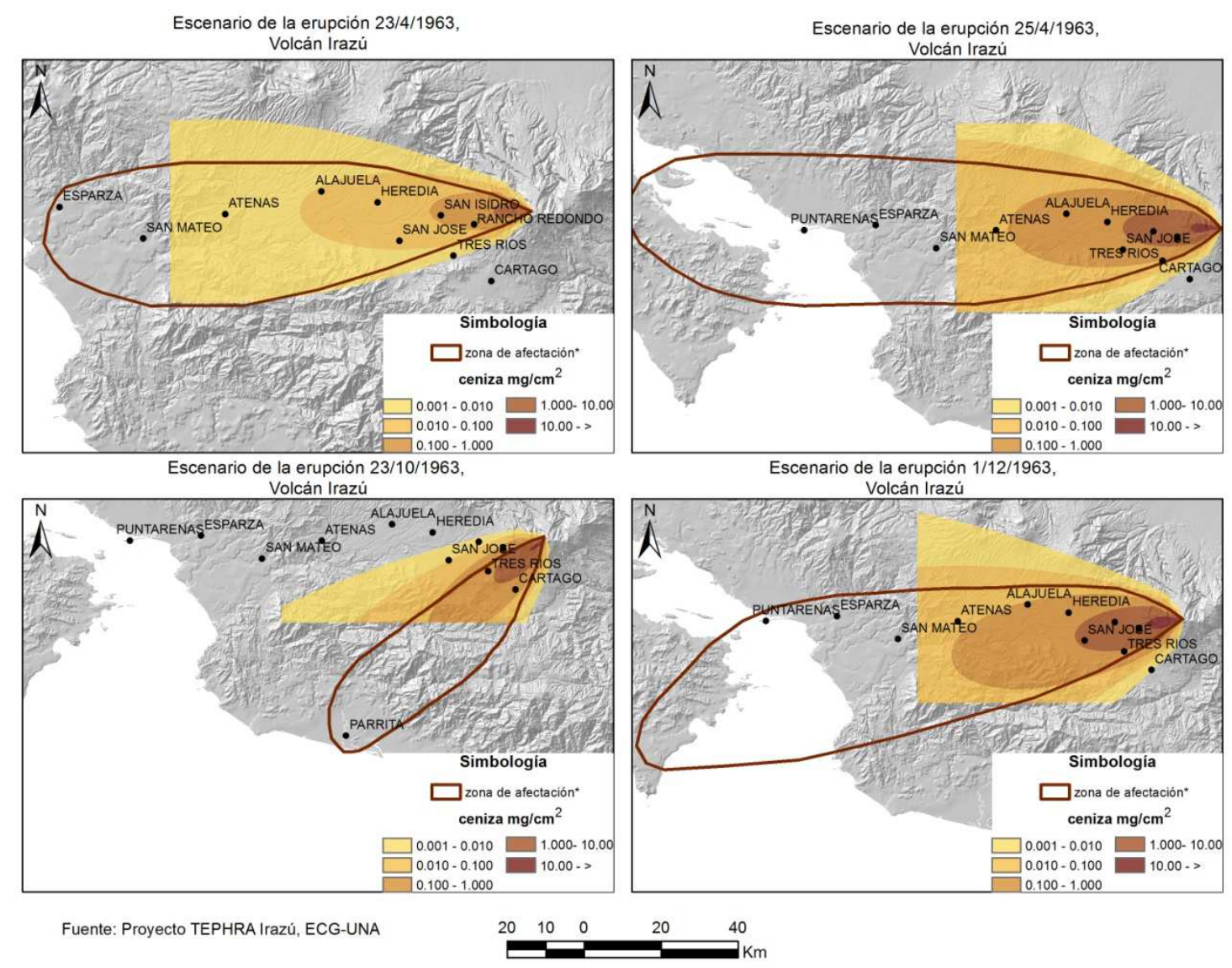

Figura 1. Escenarios construidos con el programa TEPHRA y su comparación con las áreas de afectación (línea café) reportadas por Barquero (1977). A corresponde al escenario de la erupción del 23 de abril de 1963. B corresponde al escenario de la erupción del 25 de abril de 1963, C corresponde al escenario de la erupción del 23 de octubre de 1963. D corresponde al escenario de la mayor erupción reportada a inicios del mes de diciembre de 1963.

Es importante aclarar que las áreas de distribución de ceniza rebasan ampliamente el área de estudio, la cual corresponde con el área de baja a muy alta afectación, por razones de tiempo y costo computacional se dejó por fuera el resto del país.

El 23 de abril de 1963 se reportó una precipitación de ceniza en lugares tan distantes como Macacona y Esparza, a más de $85 \mathrm{Km}$ de distancia (Figura $1 \mathrm{~A}$ ). Si bien, el escenario construido no abarca toda esta zona, es claro que ampliando el patrón observado, la ceniza alcanzaría a cubrir estas localidades con una fina capa de ceniza. Por otro lado, los reportes periodísticos de

Geo UERJ - Ano $15, n^{\circ} .24$, v. 1, $1^{\circ}$ semestre de 2013 p. 5-17

ISSN: 1415-7543E-ISSN: $1981-9021$

http://www.e-publicacoes.uerj.br/index.php/geouerj 
la época señalan que la zona de mayor afectación, luego de este episodio, se extendió hasta Rancho Redondo (Periódico La Nación, 1963), poblado que según la simulación habría recibido alrededor de $0.18 \mathrm{~cm}$ de ceniza en esta erupción (asumiendo una densidad de $1,2 \mathrm{~g} / \mathrm{cm}^{3}$ ).

El 25 de abril se reporta una gran cantidad de erupciones de las cuales sobresalen dos erupciones importantes, la primera con una columna eruptiva de alrededor de $6700 \mathrm{~m}$ y la otra de $8000 \mathrm{~m}$ (Periódico La Nación, 1963). Los poblados para los que se reporta caída de ceniza son: San José, Heredia, Alajuela, San Ramón, Puntarenas y se indica que la ceniza alcanzó incluso Guanacaste (Figura 1 B). Para este suceso se modeló únicamente el evento mayor, de acuerdo con la simulación, sobre Rancho Redondo se depositarían alrededor de 2,8 cm, en San José 0,4 cm y en Heredia 0,3 cm. Como puede apreciarse en la figura $2 \mathrm{~B}$, la tendencia general en la distribución de la ceniza se aproxima a la delimitación realizada Barquero (1977) con base en la información periodística.

El 23 de noviembre se produjo una erupción grande, inusualmente la direccción del viento cambió, dirigiendo la ceniza hacia el suroeste. Como resultado se reporta abundate ceniza sobre la Ciudad de Cartago, incluso se reporta paralización del tránsito a la altura de Ochomogo (Periódico La Nación, 1963) por la intensidad de la caída de ceniza. De acuedo con los resultados del modelo sobre Cartago cayeron alrededor de $2,5 \mathrm{~cm}$ de ceniza y la tendencia sugiere que la ceniza alcanzaría Parrita como lo reportó Barquero (1977).

Entre el 1 y el 9 de diciembre se produjeron una serie de erupciones, que no se distinguen en los registros históricos o periodísticos disponibles, debido a que en los primeros días del mes ocurrieron las erupciones más violentas. De acuerdo con Barquero (1977) los vientos llevaron la ceniza hacia el oeste, alcanzado incluso el poblado de Cóbano, en la península de Nicoya, y en esta ocasión se reportó una gran afectación sobre San José. Nuevamente, solo se trató de modelar el evento mayor en el Valle Central, que se representa en la figura 1D, según este escenario sobre San José habrían caído alrededor de $2 \mathrm{~cm}$ de ceniza. 
Como se puede deducir de los escenarios reconstruidos, la aplicación del programa de simulación TEPHRA modificado, desarrollado a partir del modelo Suzuki permite una razonable reproducción de los escenarios de erupciones documentadas para el período de actividad 63-65.

\section{ANÁLISIS DE TENDENCIAS}

En el apartado anterior se evaluaron escenarios individuales para comparar cualitativamente su aproximación a lo ocurrido, en este apartado el análisis se centra en la valoración de las tendencias generales, como medio para evaluar la calibración realizada.

La primera aproximación se realizó comparando la ceniza acumulada en 52 escenarios de distintas intensidades (VEI I, II y III), la figura 2 muestra la comparación de ésta ceniza acumulada y las isopacas del periodo eruptivo 1963-65 levantadas por Clark (1993), en la figura se puede apreciar un buena correspondencia entre la extención e intensidad de la ceniza acumulada con respecto a las isopacas. No se comparan los montos y altura de la tefra porque estas corridas del modelo no emulan todas la erupciones ocurridas durante este periodo.

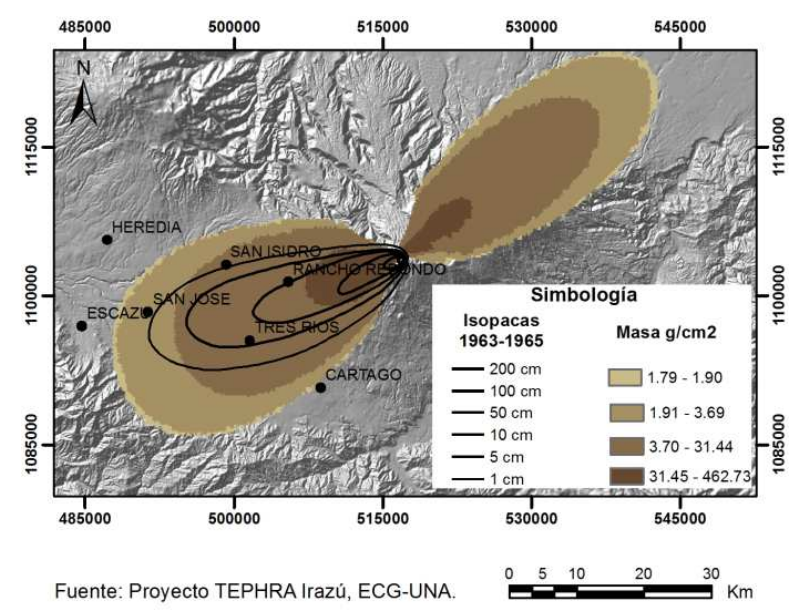

Figura 2: Comparación entre masa acumulada por 52 simulaciones, con la distribución de las isopacas del periodo eruptivo 1963-65, según Clark (1993).

La diferencia con respecto a las isopacas de Clark es un leve desvío al sur oeste por parte del modelo, la diferencia pude explicarse en razon de que los 52 escenarios sumarizados en la figura 
3 consideran al menos las tres principales direcciones del viento y no lo ocurrido presisamente entre 1963-64, debido a la ausencias de registros de viento para éste periodo. Clark, Reagan y Trimble (2006) notan que las elipses de las isopacas de erupciones ocurridas durante los últimos 2600 años (en su mayoría pequeñas erupciones) tienden a coincidir con el eje de la cuenca superior del río Reventado, en tanto, las elipses de las isopacas de las erupciones más grandes, ie. 1963-65 o 1723, tienden a ubicarse un poco más hacia el suroeste (ver contraste entre las isopacas de las figuras 2 y 3). Obviamente, la masa acumulada por 52 simulaciones (figura 3) estaría controlada por las erupciones más voluminosas que según Clark, Reagan y Trimble (2006) tienden a distribuirse un poco más hacia el suroeste que las erupciones más pequeñas, como ocurrió en el período 1963-65.

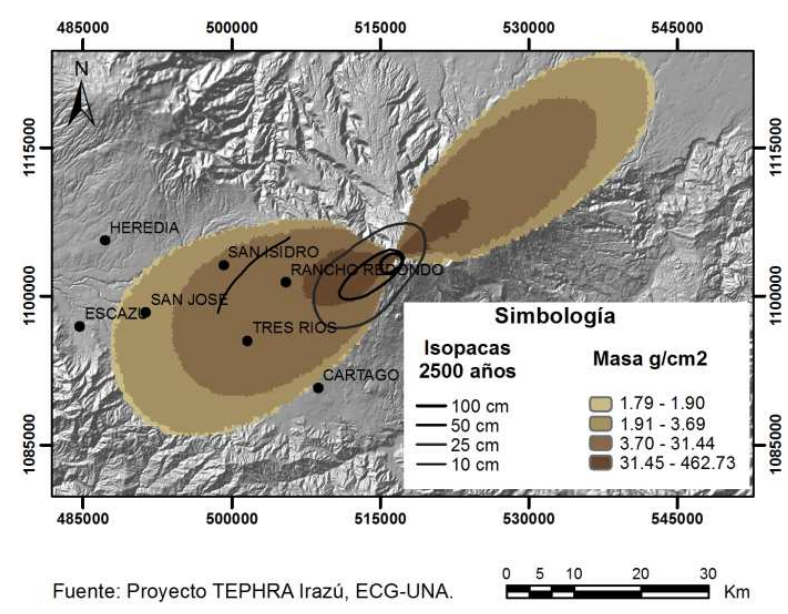

Figura 3. Comparación entre masa acumulada por 52 simulaciones, con la distribución de las isopacas de los últimos 2600 años, según Clark (1993) y Clark, Reagan y Trimble (2006).

Otro aspecto que llama poderosamente la atención es el lóbulo de ceniza que se extiende hacia el sector noreste del cráter, sin embargo, Alvarado (2000) ya había registrado esta tendencia en el mapa generalizado de distribución de ceniza para el período 1963-65 (Figura 4). También, al observar la distribución de las isopacas correspondientes a los útimos 2600 años (Clark, 1993; Clark, Reagan y Trimble, 2006) se denota la presencia de la isopaca de $25 \mathrm{~cm}$ hacia el noreste del 
cráter, tendencia que indica que no es casual dicha acumulación que el modelo reproduce al utilizar las direcciones de viento más probables en las diferentes capas altitudinales.

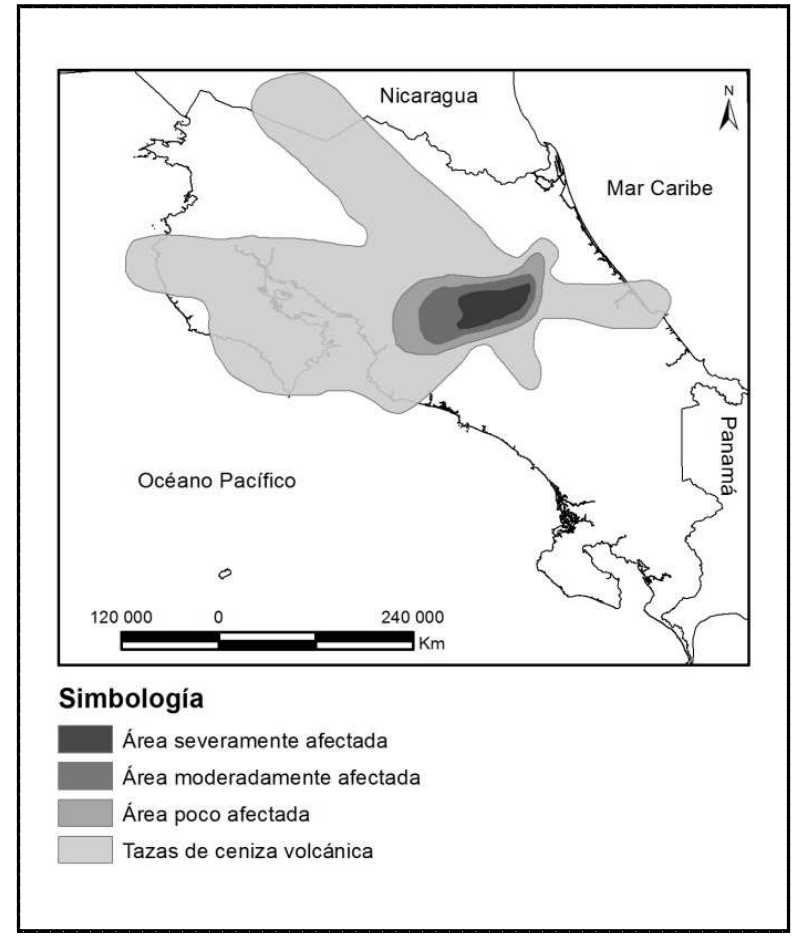

Figura 4. Mapa generalizado de la distribución de ceniza del período activo 63-65 del volcán Irazú según Alvarado (2000).

El buen desempeño mostrado por los escenarios individuales y acumulados, indica que el programa TEPHRA modificado es apropiado para la construcción de mapas probabilísticos en los que se considere la probabilidad de los escenarios. Estos mapas son de gran utilidad en la planificación del ordenamiento territorial.

\section{CONCLUSIONES}

La simulación computacional es una poderosa herramienta para simular el comportamiento de los sistemas naturales, particularmente útil en el caso de la amenaza por caída de ceniza, al permitir reconstruir las áreas y grados de afectación en caso de una erupción.

El modelo Suzuki implementado por medio de la modificación y ampliación del programa TEPHRA realiza una buena aproximación de la deposición de ceniza para los episodios eruptivos mejor documentados del período eruptivo 63-65 del volcán Irazú, dada la similitud 
entre los registros históricos y las áreas de afectación reconstruidas utilizando el modelo. Una calibración más precisa del programa sería posible de conocerse mejor las áreas de afectación para eventos selectos durante el periodo eruptivo en consideración.

Al comparar las tendencias generales de 52 simulaciones que contemplan varios niveles de intensidad volcánica, contra los mapas de isopacas para el episodio eruptivos 1963-65 y para los depósitos de tefra acumulados durante los últimos 2600 años se nota un acople satisfactorio, e indica tendencias que han sido pasadas por alto en otros trabajo. Esta situación justifica la aplicación del programa TEPHRA modificado en la elaboración de mapas de amenaza por caída de ceniza para el Volcán Irazú y otros volcanes.

\section{REFERENCIAS}

Alvarado, G. E. (1993). Volcanolgy and petrology of Irazú Volcano, Costa Rica. (Tesis Doctora no publicadz). Universidad de Kiel, Alemania.

Alvarado, G. (2000). Los volcanes de Costa Rica: su geología, historia y riqueza natural (2 ed.). San José:EUNED.

Alvarado, L. F., y Fernández, W. (2001). Climatología de la Atmósfera Libre sobre Costa Rica. Top.Meteor. Oceanog, 8 (2), 89-115.

Armbrister, T. (1964). The sky is falling. Saturday Evening Pos, 237, 20.

Armienti, P., y Macedonio, G. (1988). A Numerical model for simulation of tephra transport anddeposition: applications to may 18, 1980, Mount St. Hellens Eruption. Jornal of geophysical research,

93 (B6), 64-76.

Astorga, A. (2008). Análisis de Alcance Ambiental del Plan GAM; Parte II. San José.

Barquero, J. (1977). El volcán Irazú y su actividad.(Tesis de licenciatura no publicada). Universidad Nacional

Barrantes, G., Segura, A., \& Walcott, K. (2009). Percepción y prevención del riesgo por lahar en LosDiques de Cartago. Revista Geográfica de América Central (42), 57-82.

Geo UERJ - Ano $15, n^{\circ} .24$, v. $1,1^{\circ}$ semestre de 2013 p. $5-17$

ISSN: 1415-7543E-ISSN: 1981-9021

http://www.e-publicacoes.uerj.br/index.php/geouerj 
Clark, S. K. (1993). The recent eruptive history of Irazú volcano, Costa Rica: a study of tephra deposits ofthe last 2,500 years with geochemical and isotopic analysis of the 1963-65 eruption. Tesis paraobtener el título de Máster of Science en Geología . Universidad de Lowa.

Clark, S.K., Reagan, M.K. y Trimble, D.A. (2006) Tephra deposits for the past 2600 years from Irazuvolcano, Costa Rica. GeologicalSocietyofAmericaSpecialPaper 412, 225-234.

Connor, C. B. (5 de Junio de 2002). Tephra dispersion modeling in parallel.Recuperado de la página de laUniversidad de Florida del Sur http://www.cas.usf.edu/ cconnor/parallel/tephra/tephra.html

Escuela Centroamericana de Geología. (1993). Amenaza y estimación económica básica de lavulnerabilidad material asociada al Volcán Irazú Cordillera Central, Costa Rica. SAN JOSÉ:CEPREDENAC.

Gómez, J. (2008). Clúster interuniversitario: herramienta para la investigación más competitiva einterdisciplinaria. Boletín de ciencia y tecnología (71).

Murata, K. J., Dondoli, C., y Saenz, R. (1965). The 1963-65 eruption of Irazú volcáno, Costa Rica (theperiod of march 1963 to octuber 1964).U.S. Geolgical Survey.Se expande zona de muerte causada por las cenizas del Volcán Irazú. (23 de Abril de 1963). La Nación ,pág. 20.

Subsecretaría de Desarrollo Regional y Administrativo de Chile (2011) Guía: análisis de riesgosnaturales para el ordenamiento territorial. Santiago, Chile: Editor.

Suzuki, T. (1983). The theoretical model for dispersion tephra. En D. Shimozuro, \& I. Yokoyama, Arc Volcanims: physic and tectonics (págs. 95-113). Tokyo: Terra Scientific publishing company.

Waldron, H. (1967). Debris Flow and Erosion control problems caused by the ash eruptions of IrazúVolcano, Costa Rica. geological survey Bulletim , 1-37.

Artigo recebido para publicação em maio de 2013.

Artigo aceito para publicação em junho de 2013.

Geo UERJ - Ano $15, n^{\circ} .24$, v. $1,1^{\circ}$ semestre de 2013 p. $5-17$

ISSN: 1415-7543E-ISSN: 1981-9021

http://www.e-publicacoes.uerj.br/index.php/geouerj 